\title{
RECHERCHE MÉDICALE EN AFRIQUE SUBSAHARIENNE : SES CONTRAINTES ET SES
} POTENTIALITÉS

DUMAS Michel 1

1. Limoges, France

E-Mail Contact - DUMAS Michel : ient (at) unilim (dot) fr

\section{RESUME}

Un nombre, non exhaustif, de constats sur la situation actuelle de la recherche médicale en Afrique subsaharienne est rapporté ; la prise en compte de ces constats pourrait en permettre un meilleur développement.

Des réflexions concernant l'organisation de la recherche, ses financements, la nécessaire sensibilisation des décideurs, le statut et le rôle du chercheur, ainsi que la structuration de la recherche, permettent de mieux cerner certains des freins actuels.

Des suggestions sont formulées ; chacune d'entre elles doit être adaptée au contexte de chaque pays. II appartient aux seuls chercheurs du Sud de prendre les décisions adéquates et de les développer en partenariat étroit et égalitaire avec d'autres chercheurs. Parmi les suggestions, citons : - la potentialisation des moyens par le développement de réels Réseaux, régionaux et internationaux, de chercheurs,

- la création, au Nord, de postes virtuels d'Attachés de Recherche à titre Etranger, - la publication d'une ou deux revues médicales africaines de haut niveau, et le développement de la banque de données africaines «lndex Medicus».

Enseignement supérieur et recherche sont indissociables, quelle que soit la matière enseignée et la recherche effectuée. Il en est bien entendu de même de la médecine et de la santé en général.

En médecine, le rôle d'un enseignant est triple; il concerne :

- la formation du personnel de la santé, et particulièrement des étudiants,

- le soin à procurer au patient,

- et enfin la recherche médicale,

- et dans ces trois domaines, en outre une implication dans des tâches administratives avec parfois de lourdes responsabilités.

II n'est pas nécessaire d'avoir le statut officiel d'enseignant pour devenir chercheur, mais il est exceptionnel que le chercheur ne participe pas aussi à l'enseignement. Une tâche plus qu'une autre peut être privilégiée, mais il est indéniable que les liens entre les trois activités sont primordiaux et que chacune d'entre elles participe à l'enrichissement continu des deux autres.

La recherche alimente l'enseignement qui devient ainsi de qualité, et la confrontation quotidienne avec le malade ne peut que susciter interrogation, avec le souci d'y apporter une réponse, donc d'organiser une ou des recherches.

$\mathrm{Ce}$ propos consacré à la recherche médicale en Afrique subsaharienne, comprendra quatre développements ; le premier sera consacré aux enjeux de la recherche médicale ; le deuxième établira un constat de l'existant ; le troisième sera consacré à des réflexions, et le quatrième à des suggestions. Ces dernières peuvent éventuellement favoriser le développement de la recherche médicale; mais, même si elles sont susceptibles de concerner tous les Etats d'Afrique subsaharienne, il ne peut s'agir que de pistes qui doivent obligatoirement être adaptées au milieu médical, universitaire, socioculturel, économique et politique de chacun des Etats, en fonction du niveau de développement de chacun d'entre eux. II n'existe aucune solution miracle et universelle, et il est bien évident qu'il appartient aux seuls chercheurs et décideurs du Sud de prendre les décisions adéquates; les chercheurs du Nord n'ont pas autorité à proposer des directives, mais seulement, en raison de leur expérience, à aider leurs collègues du Sud à trouver les solutions les mieux adaptées pour résoudre chaque problème dans leur contexte, sans chercher à copier ce qui existe en Occident, mais à adapter des propositions à la culture et aux possibilités locales. 


\section{I - Enjeux de la Recherche}

Ces enjeux sont primordiaux ; ils sont intellectuels, pédagogiques, économiques, politiques et culturels :

- intellectuels, car la recherche développe l'aptitude à la compréhension et à l'interprétation des faits ; ceux-ci, même s'ils sont souvent évidents, peuvent échapper à la perspicacité d'un observateur, non chercheur. Etre chercheur, c'est être curieux ; c'est acquérir la capacité de l'analyse, de la réflexion, de la transposition, de l'adaptation du contenu d'une recherche au développement sanitaire de son pays;

- pédagogiques, car comment envisager un enseignement de qualité s'il n'est pas sous tendu par une recherche elle-même de qualité. L'esprit de recherche se transmet à travers l'enseignement ; le chercheur devient un modèle pour les jeunes générations ; l'enthousiasme qu'il manifeste à travers sa Recherche suscite des vocations. Sans esprit de recherche, l'enseignant peut devenir un perroquet, tout juste capable d'ânonner les découvertes d'autrui. Son enseignement devient rapidement un ballon de baudruche de niveau discutable, qui de toutes manières ne sera jamais un enseignement d'excellence ; - économiques, car toute recherche peut être valorisée ; ceci est particulièrement vrai dans le domaine thérapeutique ; les retombées économiques peuvent parfois être majeures ; certains aspects de la médecine traditionnelle ne sont pas tous exploités ;

- politiques : car la recherche est un facteur d'autonomie dans le développement. L'absence de recherche propre maintient une dépendance avec autrui ; il est impossible d'acquérir une reconnaissance et une vraie liberté : la liberté du choix décisionnel, pour orienter sa politique de développement médical. La maîtrise de la Recherche médicale permet l'accession à cette vraie liberté, et au respect manifesté par autrui ;

- culturels : il s'agit probablement du principal enjeu. La recherche est indissociable de la culture qu'elle prétend servir; celle-ci, comme un pont qui s'élance d'une rive à l'autre, s'appuie sur des piliers profondément enfouis dans le sein d'une nation. Comment envisager une recherche médicale qui ne s'appuie pas sur la culture d'une nation ? Comment concevoir qu'une recherche médicale de qualité puisse répondre aux aspirations légitimes d'un peuple si elle ne trouve pas son fondement dans la culture même de ce peuple? La culture, qui permet de comprendre et d'interpréter le rôle joué par les facteurs environnementaux auxquels la médecine est profondément liée ; comment interpréter le rôle bénéfique ou maléfique de ces multiples facteurs environnementaux: alimentaires, climatiques, toxiques, vectoriels, économiques, sans référence à sa propre culture ? Culture, qui constitue le véritable noyau d'une société qui permet à l'être de s'identifier et dans laquelle il puise force et vitalité ; culture qui est source de vie, et dont la privation explique le déracinement des êtres expatriés ; culture qui est source de bien-être, d'harmonie, de stabilité, et dont le rejet va occasionner au mieux de simples déboires, mais le plus souvent une perturbation de la santé de l'individu. La santé est en effet indissociable de la culture. La non prise en charge de cette dimension conduit à l'échec de toute action de santé préventive et curative où que ce soit ; ceci est d'autant plus flagrant et grave que le pays a un retard dans l'organisation de ses services de santé. La perception de cette dimension culturelle peut et doit conduire à une réflexion, et si cela est nécessaire, à une révision des actions médicales.

L'ensemble de toutes ces considérations nous permet de comprendre : - d'abord que la recherche médicale est primordiale, qu'elle est une nécessité et qu'il ne peut y avoir de développement durable d'une nation sans la prise en compte de cette composante. Ce développement durable est tributaire du bon état de la santé physique et morale des individus qui sont à la fois acteurs et bénéficiaires du développement, d'où l'importance de l'enjeu ;

- qu'ensuite elle ne peut réellement se développer que dans le contexte qui a suscité l'interrogation du soignant, de l'enseignant, du chercheur, c'est à dire au sein de la société où elle trouvera son application.

\section{II - Constat Actuel}

Dès lors, on doit s'interroger sur le niveau du développement de la recherche médicale en Afrique subsaharienne, sur ses déficiences, et même parfois sur la réalité de son existence et sur les conditions propices à son développement, et si cela est le cas, à son émergence.

Une analyse du développement de l'enseignement médical supérieur, creuset de la recherche médicale, permet de souligner les nombreuses réalisations effectuées depuis un demi-siècle; les plus remarquables sont :

- l'émergence de multiples institutions d'Enseignement Supérieur Médical dans la majorité des pays d'Afrique Noire francophone ;

- la formation de nombreux médecins, de spécialistes et d'enseignants- chercheurs ;

- l'organisation du Conseil Africain et Malgache pour l'Enseignement Supérieur (CAMES);

- la promotion et la nomination d'enseignants-chercheurs sous le couvert de ce CAMES ;

- l'émergence du Réseau de la Conférence Internationale des Doyens de Médecine d'Expression Française (CIDMEF) et de quelques Réseaux de spécialistes (neurologues, radiologues...); - la mobilité des étudiants et des enseignants-chercheurs ;

- la formation doctorale, la co-diplômation ... 
On peut donc estimer que toutes les conditions requises pour un plein épanouissement et un plein développement de la recherche médicale existent.

Pourtant, malgré ce terrain propice souvent excellent, les résultats ne sont pas à la hauteur de l'attente et des enjeux ; les déficiences et les échecs sont multiples ; on constate :

- un mauvais rapport coût-bénéfice ;

- un faible ancrage de l'Université Médicale et de la Recherche dans le monde économique et social du pays, en particulier dans le monde rural ;

- un exode des compétences vers l'Occident ;

- un mauvais usage et un éparpillement des moyens, aggravé par un cloisonnement des activités de recherche à l'intérieur d'un même pays et, à plus forte raison d'un pays à un autre, dans une même sousrégion géographique.

En définitive, il y a une absence de développement significatif de la recherche médicale, qui se traduit par la non émergence ou l'extrême rareté des Centres d'Excellence de Recherche, qui avaient constitué une des pierres angulaires du développement de la coopération scientifique soutenue par les autorités européennes, et françaises en particulier.

II paraît donc nécessaire, si ce n'est indispensable, d'analyser sans concession les causes de ce qui peut parfois être considéré comme un échec, afin de d'envisager d'éventuelles solutions. La situation est bien entendu différente et inégale selon les pays ; mais d'une manière générale, le constat global est semblable ; partout, il traduit le mauvais développement de la recherche. Nous soulignerons quelques constats, dont la prise en compte pourrait probablement permettre d'être plus efficace.

Un premier constat, contrairement à ce que l'on pourrait penser, est que la recherche est rarement prise en compte par les organismes d'enseignement supérieur médical, et ceci même si souvent la dénomination du Ministère de tutelle de ces organismes, juxtapose la double proposition de l'enseignement et de la recherche. La recherche relève encore trop souvent du seul fait individuel et ponctuel d'enseignants qui, avec peu de moyens, effectuent une recherche essentiellement clinique, parfois épidémiologique, et plus rarement, si ce n'est exceptionnellement fondamentale. L'Université demeure trop souvent absente ou en retrait, et ceci même si de nombreux enseignants-chercheurs ont acquis des Diplômes d'Etudes Approfondies (DEA), des Maîtrises et des Doctorats attestant de leur compétence, de leur qualité de chercheur, et de leur capacité à conduire d'excellentes recherches. Les conditions d'exercice de la recherche médicale ne constituent pas toujours un réel objectif clairement affiché et soutenu dans les Universités. Paradoxalement, les chercheurs universitaires sont rarement ou insuffisamment associés à un des grands organismes mondiaux de recherche, lorsque ceux-ci existent dans le pays, comme les Instituts Pasteur ou l'Institut de Recherche pour le développement (IRD) dans les pays francophones; la recherche y est encore essentiellement conduite par des chercheurs du Nord.

Un deuxième constat est que le chercheur a rarement sa véritable place dans la Nation, où il est trop souvent considéré comme un véritable sous-prolétaire, et non pas comme une des forces vives, productrices, de la Nation, au même titre que les responsables dans les secteurs de l'économie. Pire, la gestion de la carrière des individus formés est trop souvent aberrante et anti-économique. Leurs formations, coûteuses et longues, au moins partiellement et souvent totalement effectuées au Nord, ne débouchent pas obligatoirement sur l'émergence de l'emploi pour lequel ils ont été préparés. De retour dans son pays, le chercheur espère et fait d'abord preuve de patience ; il attend longtemps mais trop souvent en vain, pour finalement soit renoncer et s'adonner à d'autres activités, soit chercher à s'expatrier sous des cieux occidentaux qui lui paraissent plus hospitaliers. Ceci traduit, de la part des décideurs, une méconnaissance de l'importance des enjeux et une absence de volonté politique de rentabiliser au mieux les moyens existant dans le pays. II en résulte bien entendu un sentiment de frustration important pour les chercheurs ; frustration de cette non reconnaissance par les autorités gouvernementales des efforts du chercheur pour l'acquisition d'une excellente formation souvent loin de sa famille et de son milieu culturel ; frustration aussi engendrée par le sentiment ressenti d'un désintérêt de la part de leurs collègues occidentaux, qui s'étonnent de l'absence de résultats ; frustration devant l'impossibilité pour la majorité d'entre eux de participer aux grandes manifestations scientifiques internationales par manque de ressources financières; nombreuses autres frustrations qui ne peuvent toutes être détaillées. II en résulte un sentiment d'abandon puis de découragement, qui favorise l'exode vers le miroir aux alouettes que représente la recherche, en apparence de luxe, pratiquée dans les pays dits nantis. On estime actuellement à 20000 le nombre d'africains professionnels de tous ordres de la santé qui émigrent au Nord, chaque année, depuis 1990 *. Les responsables des pays occidentaux ont eux-mêmes une politique ambivalente et coupable, prônant à la fois le développement des pays mal nantis, en contribuant à la formation de leurs élites, tout en favorisant ensuite, dans un véritable détournement intellectuel, leur intégration en Occident dans des équipes hospitalières et de recherche, réduisant ainsi à néant tous les efforts mis en place depuis de nombreuses 
années pour favoriser le Développement Durable d'une Nation.

Un troisième constat est la difficulté qu'éprouvent les chercheurs du Sud à promouvoir les résultats de leur recherche dans la grande presse médicale internationale, ceci pour de multiples raisons, très souvent inhérentes à des questions de forme dans la rédaction des articles, et au désintérêt qu'éprouvent les rédacteurs en chef des revues occidentales, pour des recherches qui ne sont pas toujours de pointe ou d'actualité et qui ne concernent pas le monde occidental. II en résulte une méconnaissance internationale des travaux des chercheurs du Sud, faisant croire à tort à une véritable absence de recherche dans les pays où elles sont effectuées.

Un quatrième constat est la difficulté qu'éprouvent les chercheurs du Sud à avoir accès à tous les moyens modernes d'information (internet, banques de données, communications satellitaires, télémédecine, VSAT ${ }^{* *}$, etc ...). Les réseaux sont souvent peu efficaces, si ce n'est inexistants, et toujours très coûteux, car non ou mal organisés par les Universités. Ceci peut paraître très surprenant en 2008, mais c'est malheureusement une réalité dont se plaignent les utilisateurs.

Un cinquième constat est la difficulté qu'éprouvent les chercheurs du Sud à obtenir des contrats de recherche ; les bailleurs de fonds, en raison de leur situation le plus souvent au Nord, ont trop longtemps mal pris en compte le fait économique, social et culturel des pays du Sud. Par ailleurs, les fonds alloués à la recherche le sont préférentiellement en faveur des trois grandes endémies sévissant au Sud (SIDA, paludisme, tuberculose) ; ceci est bien entendu justifié, mais il faut considérer qu'en fait, ces endémies intéressent aussi et au plus haut point les pays du Nord qui se sentent menacés par ces affections. II est par contre exceptionnel que les fonds soient alloués à la promotion de recherches dont l'intérêt est exclusivement ou au moins essentiellement en faveur des pays du Sud ; ce qui est le cas par exemple pour certaines parasitoses comme la trypanosomose humaine africaine, affection mortelle encore traitée, dans sa composante neurologique, par un médicament arsenical toxique, responsable chez 5 à $10 \%$ des patients, d'une encéphalopathie mortelle. Le partenariat Nord-Sud, si souvent prôné, n'est pas encore un partenariat suffisamment égalitaire; détentrices des moyens financiers, les nations du Nord imposent encore trop souvent leurs vues, malgré le souhait clairement exprimé par les chercheurs du Sud, de ne plus être considérés comme simples spectateurs de leur recherche, simples prestataires de services, mais comme de véritables acteurs.

Un sixième constat concerne les pratiques réglementaires pour la mise sur le marché d'un médicament ou de tests diagnostiques actuellement en vigueur dans les nations du Nord; ces pratiques ne sont malheureusement pas adaptées à la situation sanitaire des pays du Sud. Les pays en développement acceptent et subissent donc les cadres réglementaires édictés par le Nord pour des populations vivant au Nord. Ces règlements interdisent la poursuite du développement de médicaments dont l'existence pourrait se révéler essentielle pour des patients vivant au Sud. L'industrie pharmaceutique, en effet, trouve rarement dans les pays du Sud, un marché suffisant pour valoriser un investissement très lourd ; les recherches thérapeutiques ne sont donc malheureusement pas orientée vers les besoins des patients du Sud. Les cadres réglementaires très contraignants ont cependant été adaptés pour faire face à la pandémie du SIDA, qui elle, n'est pas limitée au Sud! Les problèmes du Sud ne sont évidemment pas ceux du Nord, et la façon de les aborder et de les résoudre ne peut bien entendu qu'être différente ; les contextes économiques, environnementaux et culturels sont différents, et les priorités du Nord ne sont pas celles des pays en développement. Les nations du Sud ont leur part de responsabilité ; elles n'expriment malheureusement pas ou très insuffisamment leur volonté d'une manière clairement affichée. Elles n'innovent pas suffisamment, et copient encore trop ce qui prôné au Nord.

Un septième constat est la difficulté qu'éprouvent les chercheurs du Sud à gérer leur agenda. La pénurie de personnel de santé engendre une surcharge de travail, qui est elle-même aggravée par des pertes de temps dues à la fréquente mauvaise organisation des secrétariats, à la difficulté de rechercher et de retrouver une documentation, et par ailleurs, souvent pour des raisons socioculturelles ou autre à l'obligation de désorganiser un emploi du temps. II faut y ajouter les charges d'enseignement et administratives, et pour la majorité des enseignants, l'obligation d'une dispersion dans des activités lucratives dans le seul souci, bien compréhensible, d'améliorer leurs revenus, en raison d'une insuffisance criarde des salaires.

\section{III - Reflexions}

Seuls les constats les plus apparents ont été relevés ; il existe bien entendu de nombreux autres freins au développement d'une recherche médicale de qualité. Discuter ces freins peut permettre une prise de conscience et un déblocage d'une situation qui a tous les atouts pour bien évoluer. 


\section{Réflexions concernant la situation économique des pays}

Une des premières grandes causes qui est un des dénominateurs communs à l'ensemble de ces pays, est bien entendu une gestion défectueuse des maigres ressources et avant tout, la Pauvreté. C'est elle qui, enfin, commence à mobiliser les Nations mondiales et qui vient de faire l'objet de discussions lors de la dernière réunion du G8 en Irlande. Cette mobilisation est encore très lente; quelques chiffres sont éloquents : la Fondation Bill et Melinda Gates, fondation privée, accorde à elle seule pour les recherches médicales concernant les pays en développement, un financement égal à l'ensemble des sommes fournies par le reste de la communauté internationale ! Cette notion de la Pauvreté est bien entendu primordiale, car il ne peut y avoir de développement conséquent de la recherche sans financement lui-même conséquent. C'est la variabilité du niveau de la pauvreté qui explique que les solutions à éventuellement proposer à tous les "pays dits en développement" ne soient pas identiques. Ce qualificatif de développement doit être discuté, cas par cas, et ne peut avoir une signification générale; à l'opposé, le qualificatif de " pays développé ", disons, mieux nanti, demanderait lui aussi à être discuté car le soi-disant développement recoupe aussi de nombreuses insuffisances très variables selon les pays. Les causes de la pauvreté des pays d'Afrique subsaharienne sont multiples, et il est difficile, pour les chercheurs, d'avoir prise sur elles, mais une mobilisation est possible ; elle est d'autant plus nécessaire qu'il semble heureusement se dessiner dans le monde une meilleure compréhension de la nécessité d'abonder la recherche et le développement médical en faveur des Nations les moins bien nanties. La gestion de ces fonds devra donc être rigoureuse et ne pas être laissée au seul choix des décideurs politiques. Entre 1986 et 2001, les ressources mondiales investies dans la recherche médicale sont passées de 30 milliards à 106 milliards de US\$, mais les progrès réalisés en faveur des problèmes de santé des populations les plus pauvres sont restés marginaux. L'essentiel de ces sommes a concerné des pathologies qui touchent des patients du Nord; $90 \%$ des sommes investies n'a concerné que $10 \%$ de la population mondiale. Sur 1393 nouveaux médicaments mis sur le marché entre 1975 et 1999, 1 \% seulement concernait des maladies tropicales et la tuberculose. Ces fonds internationaux, même s'ils demeurent insuffisants, sont en progression ; ils le seront d'autant plus que les chercheurs du Sud montreront leur capacité à être de véritables gestionnaires, attentifs et économes des maigres subsides qui leur sont alloués. Les fonds ne peuvent être attribués qu'en réponse à une demande précise et rigoureuse, correspondant à des projets ayant des objectifs précis et une méthodologie très clairement exposés, permettant d'atteindre les objectifs fixés. La rédaction de la fiche financière d'un projet nécessite beaucoup d'attention et de précision ; elle relève encore trop souvent de l'amateurisme, qui explique souvent que le projet, malgré son intérêt, soit purement et simplement rejeté. Le bailleur de fonds veut savoir avec précision à quoi va servir l'argent investi, et quelles en seront les retombées prévisibles. On ne devient pas chercheur pour améliorer indirectement ses revenus mensuels. Ceci étant dit, tout travail mérite salaire ; une rétribution pour les chercheurs doit pouvoir être prévue dans les projets ; en contrepartie, le chercheur doit s'impliquer personnellement et consacrer le temps nécessaire à la conduite de la recherche ; un projet de recherche n'est pas fait pour acquérir pour un usage essentiellement personnel, un véhicule tout terrain ou un ordinateur ... La compétition est très dure ; il faut apprendre à être parmi les meilleurs ; à ce titre, il est significatif de constater que parmi 1500 projets concernant la santé dans les pays en développement soumis à la Fondation Bill et Melinda Gates par 400 équipes provenant de 75 pays, seulement 43, dont un du Ghana et un du Kenya, ont été retenus dans un premier choix, et que finalement, 22 seulement ont reçu un financement !

\section{Réflexions concernant la sensibilisation des décideurs aux enjeux du développement de la recherche médicale}

Les décideurs politiques, administratifs, universitaires, ont trop souvent une mauvaise perception des enjeux, donc du rôle majeur joué par la recherche médicale dans le développement d'une nation. Tous les lobbies et canaux doivent être explorés et activés par les chercheurs pour sensibiliser les décideurs. II s'agit d'une action de volonté et de mobilisation de chaque instant. L'action est longue et certes difficile, mais réalisable, ceci d'autant plus qu'il existe actuellement dans le monde, et en France en particulier, une prise de conscience salutaire. II suffit d'être soi-même convaincu et de favoriser l'organisation de multiples réunions, de conférences de tous ordres consacrées à la recherche médicale. Sensibiliser les décideurs universitaires ne doit théoriquement présenter aucune difficulté, mais il faut avoir réellement conscience qu'une recherche qui se déroule dans et grâce à l'Université, doit avoir des retombées pour cette Université ; elle doit aussi en tirer un bénéfice, ne serait ce que dans le rehaussement de son prestige. Cela suppose que dans tout projet de recherche, dans toute publication, il y ait très clairement mentionné le nom de cette Université, de la Faculté de Médecine, et du département ou support de la recherche. Un chercheur appartient à un corps ; il doit d'autant moins l'oublier que c'est justement en raison de cette appartenance que très souvent, il y a acceptation du projet par le bailleur de fonds. La seule réputation du chercheur ne suffit pas. Les retombées doivent de même être financières. Une Université a des frais fixes ; elle apporte sa caution, sa structure, ses locaux, ses laboratoires; il est donc normal qu'elle perçoive en retour un coefficient à déterminer selon 
l'importance du projet. Cette pratique est habituelle dans les Universités du Nord. La gestion de ces fonds ne doit bien entendu pas échapper à la rigueur comptable, qui ne doit cependant pas constituer une entrave au bon déroulement de la recherche, comme cela est malheureusement trop souvent le cas.

\section{Réflexions concernant le chercheur}

Le statut du chercheur, son rôle, sa place, sa reconnaissance au sein de la Nation, doivent être mieux définis pour éviter qu'il ne soit rapidement envahi par un sentiment de dévalorisation et de frustration, qui le conduit soit à l'abandon, soit à un exode vers l'Occident. Mais, ne s'intitule pas chercheur qui le désire. Le titre de Professeur n'équivaut pas obligatoirement à celui de chercheur, mais témoigne simplement d'une certaine aptitude qui demande à être développée. II faut tout d'abord en avoir un réel désir, avoir des capacités de curiosité, d'émerveillement, être capable d'aménager son temps de travail, et avoir des qualités d'organisateur, de gestionnaire, de décideur. II faut apprendre ce métier. Une recherche ne s'effectue pas seul dans son coin, mais au sein d'une Equipe, même si celle-ci est minime ; elle ne peut s'effectuer qu'en partenariat d'abord avec ses collaborateurs propres, puis avec ses collègues au sein de l'Institution, puis avec d'autres chercheurs ailleurs. II faut tout d'abord apprendre à rédiger un projet de recherche avec une très grande rigueur, c'est à dire se former à cela; toute bonne rédaction ne peut être effectuée qu'en partenariat. La liste des recommandations est longue. Chaque objectif doit être bien pesé, réfléchi et réalisable dans les temps déterminés. Les financements doivent être parfaitement justifiés et la méthodologie parfaitement adaptée aux objectifs. Sans cette rigueur obsessionnelle, aucun projet ne peut aboutir. Tout doit être précisé : qui fera quoi, quels articles seront rédigés, où seront-ils publiés, quels en seront les auteurs et dans quel ordre? Tout doit être décidé à l'avance, en valorisant les jeunes. Le patron valorise son Laboratoire en étant le dernier signataire. Les jeunes ont besoin d'être promus et leur travail reconnu ; ils sont en tête. Les collaborateurs qui n'ont pas travaillé n'ont pas à être co-auteurs. Les bailleurs de fonds étudient tous ces aspects. Les comptes-rendus et les articles scientifiques doivent être soigneusement rédigés et en temps voulu. C'est le prix à payer, par les chercheurs, pour valoriser leur image et leur prestige, non seulement auprès de leurs pairs, mais aussi et surtout, auprès de la communauté nationale et internationale.

\section{Réflexions concernant la structuration de la recherche}

Toute recherche médicale doit pouvoir être proposée et conduite; elle se doit d'être universelle, intéresser tous les domaines de la médecine, et concerner aussi bien l'étude des mécanismes et des conséquences des maladies, que la recherche clinique, thérapeutique ou en santé publique. Dans le monde tropical actuel, elle doit avant tout être pragmatique et viser à procurer, le plus vite possible, le mieux être de l'Homme qui vit dans des régions tropicales, c'est à dire de l'Homme qui est encore trop souvent exclu des circuits mondiaux de la santé ; elle ne peut que s'atteler à défricher le vaste champ des inconnues de la pathologie qui règnent dans ce monde tropical, pour viser à procurer le bien être physique et moral de l'homme, c'est à dire le maintenir dans la plénitude de sa santé au milieu d'un environnement trop souvent défavorable. II paraît nécessaire de favoriser l'émergence de recherches qui prennent en compte la globalité des différents aspects du développement sanitaire, celles axées vers le tissu socioculturel des populations, celles en santé publique concernant les études épidémiologiques, la prévention des maladies, les inégalités, les risques sanitaires, ainsi que le fonctionnement des systèmes de santé, c'est à dire celles qui conditionnent la politique de santé publique d'une nation, donc le choix politique. Les recherches doivent aussi, et c'est évident, concerner les principales endémies, mais aussi la chaîne alimentaire, l'accès aux médicaments, l'éducation sanitaire des populations, les thérapeutiques traditionnelles, le monde du travail, l'environnement, pour ne citer que quelques une de ces thématiques, sans exclusion des autres thèmes transversaux. Ceci devrait pouvoir se réaliser par l'organisation et la promotion par les chercheurs, d'une multiplicité de microprojets regroupés au sein de thématiques unificatrices, concourant toutes à atteindre un même grand objectif. Pour atteindre ces objectifs, la recherche doit sortir des Universités où elle sera initiée, pour aller au devant du monde rural, répondant ainsi à l'attente réelle des populations qui se sentent souvent délaissées. II paraît en outre indispensable que l'organisation de la recherche soit communautaire, qu'elle implique de nombreux acteurs du corps médical mais aussi des acteurs non médecins qui peuvent apporter un savoir et un éclairage différent : architecte, agronome, sociologue, économiste, vétérinaire, démographe, géographe, anthropologue, ethnologue, etc... C'est un réel devoir pour les chercheurs tropicaux de travailler dans ces directions. En effet, qui s'intéressera à ce type de recherche, si ce ne sont d'abord et avant tout les chercheurs réellement concernés, c'est à dire les chercheurs du Sud ? Ils ne peuvent attendre que d'autres les conduisent à leur place; c'est d'abord à eux de définir les priorités spécifiques de la région dans laquelle ils vivent, et d'essayer d'apporter une réponse aux multiples inconnues qui existent encore. Les chercheurs du Sud ne peuvent plus attendre qu'on leur impose une recherche ; ils doivent être acteurs : acteurs de leur recherche, acteurs de leur développement, comme cela est prôné dans le Nouveau Partenariat pour le Développement de l'Afrique (NEPAD) ${ }^{* * *}$. 


\section{Réflexions concernant la thérapeutique}

Les recherches concernant la thérapeutique s'inscrivent dans ce contexte, y compris celles concernant la médecine traditionnelle. II est de bon ton de la valoriser, mais qu'entend-on par médecine traditionnelle ? C'est un fourre-tout, qui va du charlatanisme à la phytothérapie. La médecine traditionnelle peut être utile, mais il faut être rationnel. II faut étudier les plantes éventuellement actives et en extraire les principes actifs. II ne faut pas déposséder le guérisseur; il faut qu'il tire un bénéfice s'il y a une découverte. II est donc nécessaire d'établir des contrats, et ne pas s'approprier indûment la pharmacopée traditionnelle. A côté de cet aspect, la médecine traditionnelle peut être utile en tant que médecine psychosomatique ou de l'âme, car reposant sur la culture dans la société dans laquelle elle s'est développée ; les sociétés et leurs habitants ont besoin de ce type de médecine ; il faut alors développer une collaboration saine avec les guérisseurs. En dehors de ces deux cadres : pharmacopée traditionnelle et médecine psychosomatique, le chercheur ne peut qu'être excessivement prudent dans une éventuelle collaboration. Quelle est la part consacrée par l'industrie pharmaceutique mondiale à la recherche de thérapeutiques visant au traitement de quelques endémies uniquement tropicales, comme les trypanosomoses? Cette part est pratiquement nulle. La découverte de nouveaux médicaments doit obligatoirement être rentabilisée, mais les potentialités économiques des pays tropicaux ne sont malheureusement pas encore suffisamment attrayantes. Les recherches concernant le SIDA utilisent, et c'est heureux, d'énormes fonds, mais il s'agit d'une pandémie mondiale, même si ce sont les pays tropicaux qui, pour le moment, payent le plus lourd tribu à cette affection ; des montants, même bien inférieurs, ne sont malheureusement pas drainés par des affections, qui ont le tort de ne pouvoir se contracter que dans des pays tropicaux. Le modèle traditionnel de développement des produits pharmaceutiques, qui nécessite une rentabilité, ne permet pas d'élaborer les outils essentiels pour lutter contre les maladies négligées. Aujourd'hui, pour développer un médicament au Nord, il faut 500 millions d'Euro ! Pour les pays en développement, « la balance coût-bénéfice ne doit plus être évaluée à Washington ou à Londres, mais mesurée auprès des populations concernées ॥ ****. II faut envisager la création d'un véritable partenariat Nord-Sud, avec financement par le Nord de projets de développement de médicaments. Les sommes d'argent dégagées dans le cadre du G8 ${ }^{* * * * *}$ et du DNDi ${ }^{* * * * * *}$ (Drugs for Neglected Diseases initiative) pourraient en partie servir à cette action; les projets de recherche doivent être régionaux, concernant ainsi des pathologies communes.

L'argent ne fait cependant pas tout; il est indispensable, mais il n'est pas suffisant. II faut aussi une volonté politique forte des pays concernés qui doivent s'organiser dans un partenariat supranational. Cette organisation doit aussi concerner les pratiques réglementaires de mise sur le marché des nouveaux médicaments, des vaccins et des tests de diagnostic pour les maladies qui sévissent essentiellement ou exclusivement au Sud, en particulier pour les maladies négligées. Celles en vigueur actuellement dans les nations du Nord, ne sont pas adaptées aux problèmes du Sud, pas plus que les réglementations concernant l'éthique médicale. L'établissement d'un nouveau cadre réglementaire pour les pays du Sud est donc une nécessité ; les nations du Sud doivent exprimer leur volonté d'une manière clairement affichée, et dégager des conditions permettant l'émergence d'une solution.

\section{IV - Solutions}

Malgré la complexité des problèmes, des propositions de solutions peuvent être suggérées ; chacune d'entre elles doit être adaptée au contexte où elle doit être appliquée, ce qui signifie qu'il n'existe pas actuellement de solution universelle, et que chacune doit être discutée et acceptée par les acteurs locaux qui auront la charge de leur développement. Leur application repose avant tout sur la volonté des uns et des autres de les voir aboutir.

Trois groupes de solutions peuvent déjà être mis en œuvre ; elles visent à fournir une réponse globale à plusieurs des constats et des réflexions développées dans les lignes précédentes, mais ne prétendent pas tout solutionner. Elle concernent : le développement de Réseaux de chercheurs, la création au Nord, de poste d'Attachés de Recherche à Titre Etranger, la création au Sud d'une ou deux revues médicales supranationales de très haut niveau, et d'une banque de données médicales.

Grâce à un rapport coût-bénéfice raisonnable, la réalisation de ces trois propositions devrait permettre de promouvoir rapidement la recherche médicale en Afrique subsaharienne.

\section{Promotion de réseaux de chercheurs}

La recherche ne peut plus être le fait d'un chercheur ou d'un petit groupe de chercheurs opérant seuls dans leur microcosme ; elle est vouée à rapidement devenir apoptotique. Elle doit se nourrir d'échanges fructueux et enrichissants sur le plan des idées, en se confrontant à autrui mais aussi savoir partager les disponibilités et les moyens dont disposent les uns et les autres. Ceci est valable partout dans le monde. II se constitue des ensembles non cloisonnés mettant en commun le savoir, le savoir-faire, et les moyens qui deviennent de plus en plus coûteux. Ce partage des idées et des moyens, pour être réellement efficient, ne peut s'effectuer 
qu'au travers de Réseaux développés d'abord dans un même pays, c'est le premier niveau. Le Réseau local, doit rapidement émerger dans des Réseaux sous-régionaux et régionaux, multinationaux, puis internationaux.

C'est le but à atteindre, tout doit tendre vers l'émergence de ces Réseaux, sources d'enrichissement mutuel. Pour cela, il faut accepter de ne pas s'enfermer dans son égoïsme, considérant que la recherche est sa petite propriété ; il faut savoir partager, dans le respect mutuel et dans l'égalitarisme. II faut savoir se parler et communiquer, et additionner les compétences et les moyens. II faut aussi savoir accepter la critique de ses pairs. Le succès est à ce prix. L'individu isolé n'est pas crédible; l'Equipe l'est peu; les Réseaux par la richesse qu'ils apportent, le deviennent. C'est leur émergence qui peut permettre de dynamiser la recherche en levant les différents freins que sont les sentiments d'isolement et d'abandon éprouvés par des chercheurs vivant dans des conditions difficiles et précaires.

Ces Réseaux donneront l'habitude d'un travail en commun, permettront d'organiser de fréquentes rencontres, si possible entre individus, mais au moins grâce aux systèmes d'information à distance (VSAT) ; ils multiplieront les échanges d'informations de tous ordres. II y aura ainsi véritablement rupture de l'isolement, et promotion d'une vie communautaire de chercheurs. Sur le plan international, il est de même indispensable de favoriser le développement de tels Réseaux entre Centres de Recherche situés dans des pays considérés comme nantis, et Centres situés dans des pays plus défavorisés, mais ces Réseaux internationaux, pour être efficaces, doivent être de dimension réduite et reposer sur un réel partenariat entre personnes ayant tissé des liens d'abord humains, puis informatiques. Les Réseaux doivent demeurer pragmatiques afin de permettre de réels échanges de tous ordres. Ils peuvent permettre d'identifier les besoins pour envisager d'éventuelles contributions qui seront adaptées à un réel besoin clairement exprimé et identifié. Ces réseaux permettront l'établissement d'un partenariat sain, égalitaire, et non pas de dépendance, qui s'exprimera dans le respect de l'autre. L'émergence de ces réseaux à un niveau international sera en outre révélatrice vis à vis des autorités politiques et universitaires du pays, qui seront ainsi invitées à mieux considérer non seulement la Recherche, mais le chercheur qui trouvera une place plus honorable dans la société.

\section{Création de postes d'Attachés de Recherche à Titre Etranger}

A travers le Réseau international, il pourrait y avoir création au Nord de postes d'Attachés de recherche à titre étranger. En dehors de périodes d'activités temporaires au Nord, ces chercheurs travailleraient dans un laboratoire du Sud où ils recevraient un salaire de la part de l'organisme de rattachement au Nord pour effectuer le travail de recherche dans leur pays. II en découlerait de multiples avantages. D'abord le renforcement de la réalité du partenariat, ensuite, l'assurance d'une formation continue pour le chercheur du Sud et enfin l'amélioration conséquente de ses revenus ; ainsi dégagé de la nécessité d'en trouver d'autres, son travail serait plus efficace. Les projets de recherche pourraient même parfois financer ce poste budgétaire, ce qui serait incitateur. Les actions de recherche en seraient renforcées par le lien créé grâce à cet Attaché de Recherche à titre étranger entre un Réseau local et régional, et un Réseau International. Ceci permettrait en particulier d'apporter une solution au non-retour vers leurs pays des chercheurs formés et à plus forte raison de freiner leur exode. La création de ces postes d'Attachés de recherche à titre étranger dans les services de recherche du Nord, peut favoriser l'objectif de développement de la recherche médicale au Sud et l'émergence de Centres d'Excellence, et ainsi aider au Développement Durable.

Le chercheur et sa recherche seraient soumis à une évaluation par expertise extérieure ; la nomination serait accordée pour une période de 5 ans, éventuellement renouvelable au vu des résultats de l'expertise. Le travail de recherche effectif permettrait l'élaboration de publications qui, d'un meilleur niveau, seraient ainsi plus aisées à faire admettre dans les journaux internationaux. Enfin, ce chercheur aurait un éventuel emploi dans un pays du Nord, s'il survenait un revers politique dans son pays (les exemples récents sont multiples : Madagascar, Côte d'Ivoire, République Centrafricaine, Burundi, Rwanda, Soudan, Irak ...) ; en effet, les enseignants-chercheurs du Sud sont parfois soumis à des difficultés réelles, mettant en jeu leur propre existence, en raison de leurs convictions politiques, religieuses et/ou de leur origine ethnique, qui elle-même se superpose souvent à des groupes politiques, et ceci même si l'enseignant-chercheur n'a pas lui-même de convictions politiques affichées. Son exil vers un pays du Nord, sans ressource financière, devient alors souvent une nécessité vitale, avec toutes les difficultés et les contraintes inhérentes à ce genre de situation.

Divulgation de la recherche Elle est souvent rapportée dans des revues mal divulguées. Les grandes banques des données mondiales (Medline et autres) ne font que rarement apparaître les articles publiés dans des revues considérées comme secondaires, sans Impact Factor (IF). II est nécessaire que les communautés mondiales concernées par les affections tropicales puissent tout de même avoir accès à cette mine de documents et d'informations, qui demeure inconnue. La création d'une ou deux revues médicales africaines de très haut niveau, avec un comité éditorial international et des rédacteurs en chef élus par leurs pairs sous l'audience du CAMES, pourrait être envisagée. Cette solution se heurte évidemment à son coût. 
Par contre, dès à présent, il est souhaitable de mieux promouvoir la Banque de Données Africaines créée par Madame Marie-Paule KABORE à l'OMS à Brazzaville, qui essaye de répertorier l'ensemble des publications médicales africaines (mémoires, rapports, thèses, publications et autres) sur le site http://indexmedicus.afro.who.int/

Pour un rapport coût-bénéfice raisonnable, la réalisation de ces trois propositions permettrait de promouvoir la recherche médicale en Afrique subsaharienne.

\section{REFERENCES}

* Renforcement des systèmes de recherche sur la santé. « Rapport Mondial sur les Connaissances pour une Meilleure Santé - renforcement des systèmes de santé », OMS, 2004, 17-23. (www.who.int/rpc/wr2004)

** Very Small Aperture Terminal

*** www.nepad.org

**** Dr Pécoul : DNDI Executive Director - www.researchappeal.org

$* * * * *$ WWW-g8.gC.Ca

$* * * * * *$ WWW.dndi.org 